of pregnancy, birth, and neonatal life-are indisputably consequences of the NTD. They are secondary to the basic defect, the NTD itself. It is difficult to see how ovopathology could possibly be the cause.

M J Seller and N C Nevin Paediatric Research Unit, Guy's Hospital Medical School, London SE1 9RT; and Department of Medical Genetics, Queen's University of Belfast, Belfast BT12 6BJ.

References

${ }^{1}$ Mills JL. Malformations in infants of diabetic mothers. Teratology 1982;25:385-94.

${ }^{2}$ Martin RA, Fineman RM, Jorde LB. Phenotypic heterogeneity in neural tube defects: a clue to causal heterogeneity. Am J Med Genet 1983;16:519-25.

${ }^{3}$ Khoury MJ, Erickson JD, James LM. Etiologic heterogeneity of neural tube defects: clues from epidemiology. Am J Epidemiol 1982;115:538-48.

\section{Attitudes of Asian families to genetic counselling}

SIR,

Several surveys have been undertaken in the United Kingdom to assess the response of the indigenous population to genetic counselling. ${ }^{12}$ While the attitudes of the London Cypriot community have also been well documented, ${ }^{3}$ there is little information available concerning how genetic services might best be tailored for the British Asian population.

With this in mind a short pilot study has been undertaken based on experiences of the first 50 Asian families counselled after the establishment of a genetic counselling service in Leicester. The survey interviews were carried out in the patients' homes by an Asian doctor (LM) fluent in the relevant languages and fully conversant with Asian customs and traditions. Twenty-nine of the 50 families agreed to participate although two subsequently failed to keep a prearranged appointment. Of the 27 participating families, 23 originated from Gujarat, two from Punjab, and two from Bangladesh. Pertinent points to emerge were as follows.

Twenty-three of the 27 families expressed satisfaction with the services offered. Two families felt that further information was needed and two couples could not specifically recall having been to the genetics clinic. Three of these four families had not received a summary letter after their clinic visit; in contrast 19 of the 23 families expressing satisfaction had received summary letters. Two families, who expressed satisfaction but had not believed the high risk which was quoted at the clinic visit, subsequently had further (fortunately healthy) children. Six couples were relieved when given a low risk and intended having more children; four couples were interested primarily in antenatal diagnosis and how this could be achieved; and three couples were deterred from further childbearing by a high risk.

None of the families expressed a preference to be seen by an Asian doctor, but three families experienced severe language problems and indicated that the presence of an interpreter would have been helpful.

Termination on the grounds of fetal abnormality was acceptable in the first trimester to all but one of the 14 Hindu and two Sikh families and in the second trimester to 13 of these 16 families. For the 11 Muslim families, termination was acceptable to only five in the first trimester and three in the second. None of the 27 families would consider AID and one Muslim couple had found the subject offensive when it had been raised at the clinic. None of the Muslim families, of whom five were consanguineous, was convinced that consanguinity was hazardous.

We present these results in the hope that they will be helpful to others. Asian patients are receptive to genetic information and are usually influenced by it. Communication is obviously important and may present considerable logistic problems, since even in the relatively small city of Leicester the Asian population uses many different languages (Gujarati, Hindi, Punjabi, Bengali, Katchi). Termination is usually acceptable to those of the Hindu faith but infrequently to Muslims. If consanguinity is proven to be deleterious in the Muslim community, considerable difficulty may be encountered in conveying this to those for whom it is relevant.

\section{Mehta and I D Young Department of Child Health, Leicester Royal Infirmary, PO Box 65, Leicester LE2 $7 L X$.}

\section{References}

1 Carter CO, Roberts JAF, Evans KA, Buck AR. Genetic clinic: a follow-up. Lancet 1971;i:281-5.

2 Emery AEH, Raeburn JA, Skinner R, Holloway S, Lewis P. Prospective study of genetic counselling. $\mathrm{Br} \mathrm{Med} \mathrm{J} \mathrm{1979;i:1253-}$ 6.

3 Modell B, Ward RHT, Fairweather DVI. Effect of introducing antenatal diagnosis on reproductive behaviour of families at risk for thalassaemia major. $\mathrm{Br}$ Med J 1980;280:1347-50.

Parietal foramina in the Saethre-Chotzen syndrome

SIR,

The interesting paper by Dr Thompson and colleagues ${ }^{1}$ describing parietal foramina in a father and son with the Saethre-Chotzen syndrome 
prompts us to report a mother and daughter who presented with very similar features (fig 1).

The 4 year old daughter, who showed moderate developmental delay, was referred because of concern over her persisting large anterior fontanelle with wide metopic and sagittal sutures. Additional findings included facial asymmetry, prominent crura of both ears, and short digits with finger-like thumbs showing impaired flexion at the interphalangeal joints.

The mother, who was $147 \mathrm{~cm}$ tall, recalled being told that her own anterior fontanelle had not closed until the age of 5 years. On examination this lady, who was of limited intellect and experienced difficulty in reading and writing, had a flat receding forehead, bilateral proptosis, right ptosis, prominent crura of both ears, short fingers, and broad halluces. Palpation of her skull revealed bilateral parasagittal parietal defects each admitting a finger tip. Skull $x$-ray (fig 2) confirmed the presence of

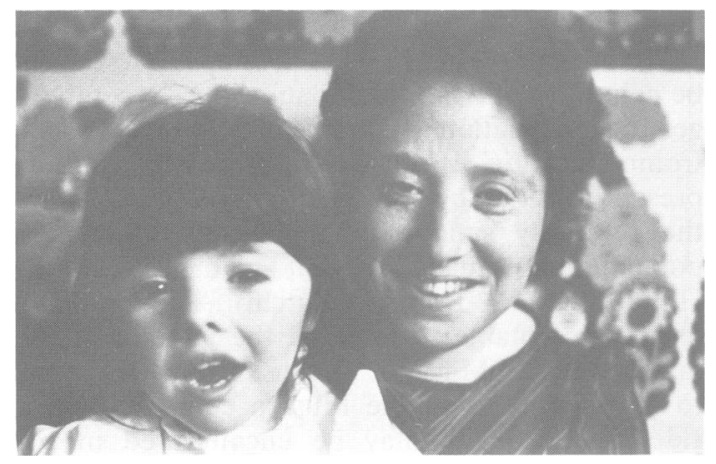

FIG 1 Mother and daughter.

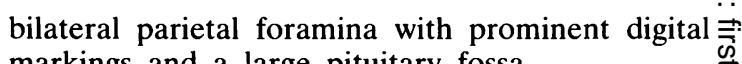
markings and a large pituitary fossa.

The findings in these patients and those of $\mathrm{Dr}$ 을 Thompson and colleagues illustrate the curious and $\frac{\bar{O}}{\bar{D}}$ important point that a disorder traditionally associ- $\frac{\sigma}{\sigma}$ ated with craniosynostosis may present paradoxi- $\varrho$ cally with delay in closure of the fontanelles and sutures. The fact that our patients showed limited $\overrightarrow{0}$ intellectual development suggests that retardation in the Saethre-Chotzen syndrome may be a primary $\vec{\omega}$ effect of the gene rather than a result of cranio- $?$ synostosis with raised intracranial pressure, as has $\overline{0}$ been suggested. ${ }^{2}$

I D Young And $P$ G F Swift or Department of Child Health, $\overrightarrow{\vec{H}}$ Leicester Royal Infirmary, $\omega$ Leicester LE2 $7 L X$.

\section{References \\ 1 Thompson EM, Baraitser M, Hayward RD. Parietal foramina $\bigcirc$ in Saethre-Chotzen syndrome. J Med Genet 1984;21:369-72. \\ 2 Pantke OA, Cohen MM, Witkop CJ, et al. The Saethre- 음 Chotzen syndrome. Birth Defects 1975;11(2):190-225.}

Philtrum length, intercommissural distance, and ear measurements in newborn infants

SIR,

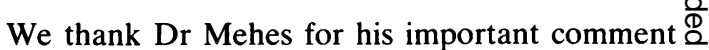
about our measurements in newborn infants. ${ }^{1} \mathrm{We} \underset{\overrightarrow{8}}{\overrightarrow{8}}$ agree indeed that all anthropometric measurements 3 should be evaluated in relation to birth weight also. $\bar{F}$ This is especially important when birth weight is not appropriate for gestational age, as in such cases

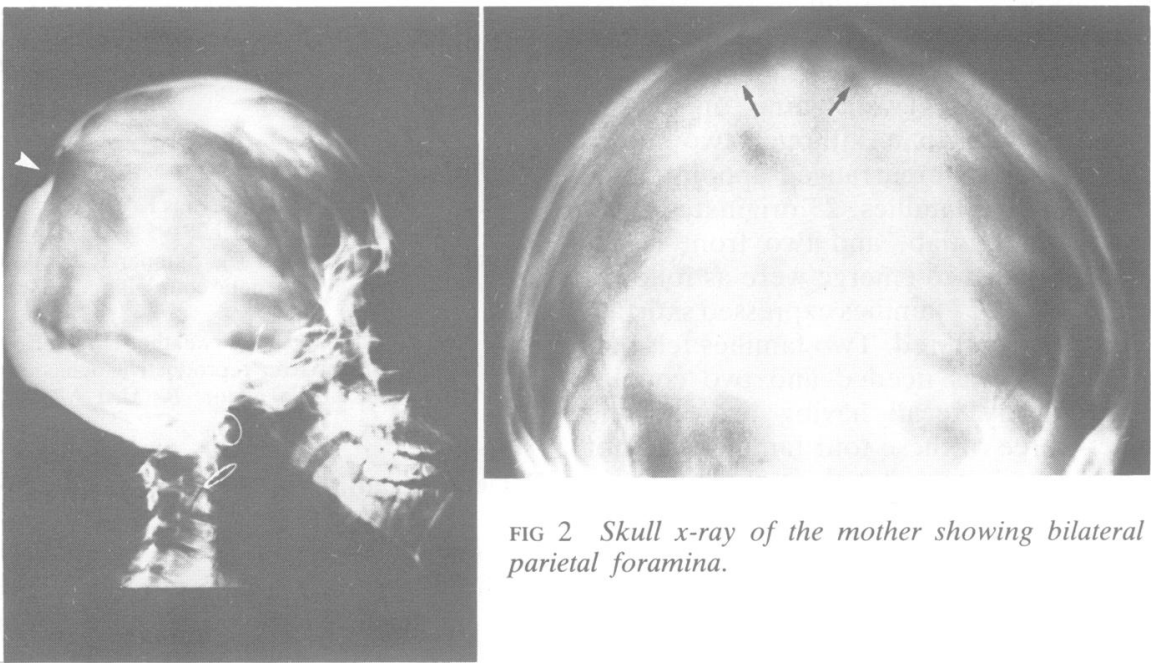

